\title{
Rapid Detection of Drugs Abuse by Adapting Surface Plasmonic Micro Ring Resonator
}

\author{
A Raganna ${ }^{1}$, Bharathi $\mathrm{S} \mathrm{H}^{2}$, Sandip Kumar Roy ${ }^{3}$ and Preeta Sharan ${ }^{4}$ \\ ${ }^{1,2}$ School of ECE, REVA University, Chennai, Tamil Nadu 603112, India; \\ ${ }^{3}$ Department of ECE, AMET University, Yelahanka, Bangalore, 560068, India; \\ ${ }^{4}$ Department of ECE, Bangalore, Karnataka, 560068, India \\ Correspondence to: Sandip Kumar Roy (sandipr@hotmail.com)
}

\begin{abstract}
Recent developments in the compact low cost optical sensors have led to a new generation of technology adaptation for low cost instruments, called lab-on-chip which are accessible for use in diverse field of science. While there are research being carried out using different field of optical sensing, current work involve Surface Plasmon Resonance (SPR). SPR is a quantum optical-electrical phenomenon that occurs as a result of the interaction of light (photons) with a metal surface (typically gold or silver). SPR based instruments for qualitative analysis has been in use. Using physical optical device which are very bulky and require skill to achieve precision in their usefulness for quantitative analysis. SPR is label-free technique hence less cumbersome and not so effort intensive compared to fluorescence-based technique. Our work is aimed at miniaturization of the bulky instrument to a nano scale level so that the same can be integrated in an integrated optical chip. As a result the device could be used extensively by patrolling police and other law enforcement agencies. We described the design and development of an optical sensor for the rapid detection of drugs abuse using the sample containing cocaine and heroin mixed in alcohol. Each constituent of the sample has unique Refractive Index (RI) and the method involves use of this unique RI as signature of the constituents of the sample. Matching of signature of cocaine and heroin in the sample confirm the presence of the drug in the sample. The final method was simulated using the proposed design parameters and sample solution of cocaine and heroin mixed in alcohol. The accuracy and reliability of the method were demonstrated using the MEEP simulation tools. The result obtained shows sensitivity of $3000 \mathrm{~nm} / R I U$ and a $Q$ factor of 2456. The result is much higher than previously published result of $250 \mathrm{~nm} / \mathrm{RIU}$ for Mach-Zehnder type optical sensor implementation.
\end{abstract}

Keywords: SPR, Biosensor, FDTD, Lab-On-Chip, Refractive Index, Drug abuse

\section{Introduction}

Tobacco, alcohol, and illicit drugs abuse is costly. As per study, more than $\$ 740$ billion annually in costs related to crime, lost work productivity and health care [25]. The most recent estimate of abuse, an increase of more than $\$ 20$ billion per year compared to six years ago [26]. Rapid detection of drug abuse is need of the hour. In a recent study [24] rapid confirmation and quantitation of drugs-of-abuse in oral fluid using a low cost, small footprint mass spectrometer is presented. However this technology involve conventional optical instrumentation setup and specialization to perform tests. Following the same principle, SPR has long been in use for quantitative and qualitative chemical analysis because of its sensitivity and robustness. SPR is one of the primary biophysical methods for the screening of low molecular weight adulterants, due to its low protein consumption and 'label-free' methodology. SPR based sensor is employed for fragment screening against drug targets. The complexity, cost and size of such instruments can often be a 
barrier to their implementation, particularly for screening applications, and their use is therefore typically limited to secondary confirmation and quantitation. The size, weight, power consumption and services required for such bulky instrumentation typically also limit their use to larger laboratories.

To address some of these issues it has long been a goal of engineers to miniaturize and simplify SPR instrumentation and thereby open up new application areas. However, each of them has their own limitations. One area where Lab-On-Chip based SPR are beginning to show real promise is when combined with integrated optics and mobile applications. Using such techniques these optical sensors have been used for a variety of rapid analysis applications such as drug detection, blood analysis and contaminations in water detection. A result of this work has been the development of an optical sensor aimed at detection of drug abuse. The proposed device size will be typically of the size of a coin.

\section{Theory}

SPR measures a change in RI of the sample over a gold plate to observe the binding interaction of sample molecules to receptor molecules immobilized on the metal dielectric interface $[14,15]$. This uses the phenomenon of SPR and the total internal reflection of light at a metal dielectric interface to generate a plasmonic wave for a limited distance of propagation path [16-18]. SPR technology were evolved since 1991 using BIAcore ${ }^{\mathrm{TM}}$ [19-21]. The basic SPR schematic is shown in Figure 1. As shown in the figure, the test surface is made up of a thin gold film on a glass prism base.

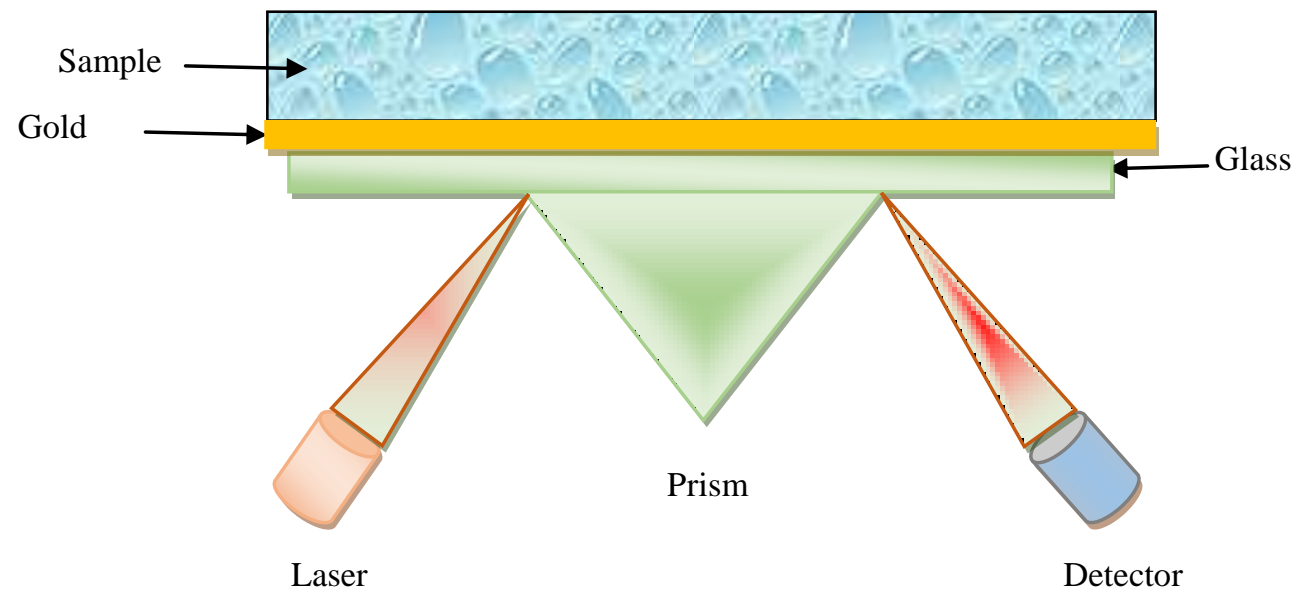

Figure 1. Diagram of the SPR Instrument Set up

The working principle of SPR remain the same in current work except the fact that instead of using big optical components we will be using optical elements in a waveguide forming part of integrated optical chip. The theory of SPR involves electromagnetic wave propagation through interface of dielectric and metal. Figure 2 provide a schematic of the boundary condition we will use to solve and structure showing the metal and dielectric interface that can support the Surface Plasmon wave. 


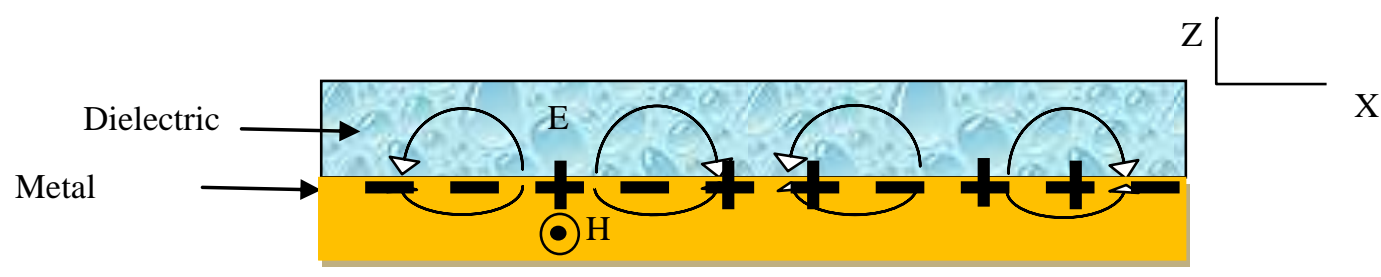

\section{Figure 2. Electromagnetic Wave Propagation through Interface of Dielectric and Metal}

When an analyte is placed in the dielectric, effective RI of test region changes. Change in RI cause change in dielectric constant $(\varepsilon)$ because

$\varepsilon=n^{2}$

Conductive materials contains large population of free electrons and the collective oscillation of these electrons are called Plasmon. When these Plasmon are confined to propagate at a metal-dielectric interface they are known to be surface Plasmon. These surface Plasmon's results in a strong electric field which is normal to the plane of interface. Coupling between the surface plasmon and light results in the Surface Plasmon Polaritons (SPP) which propagates along the interface of two medium [5].

The dispersion relationship is expressed as in Equation 2.

$$
\beta=K_{0} \sqrt{\frac{\varepsilon_{m} \varepsilon_{d}}{\varepsilon_{m}+\varepsilon_{d}}}
$$

Where, $\beta$ - Propagation constant, $\mathrm{K}_{0}$ is the wave number of light. $\varepsilon_{\mathrm{d}}$ and $\varepsilon_{\mathrm{m}}$ are permittivity of dielectric and metal respectively. The relation between real $\left(\varepsilon_{\mathrm{m}^{\prime}}\right)$ and imaginary $\left(\varepsilon_{\mathrm{m}^{\prime \prime}}\right)$ parts of $\varepsilon_{\mathrm{m}}$ is given in Equation 2. Change is wave propagation is measured by shift in frequency $(\omega)$.

When two mediam $\varepsilon_{\mathrm{d}}$ (dielectric) and $\varepsilon_{\mathrm{m}}$ (metal) involve in wave propagation then subjected to meeting boundary condition for Maxwell's equations of wave propagation is given in Equation 3.

$$
\omega^{2} \approx \frac{\omega_{p}^{2}}{2}\left(1-\frac{1}{4}\left(\frac{\omega_{p}}{c k_{x}}\right)^{2}\right)
$$

Where, $\omega_{p}^{2}=\mathrm{n} e^{2} / \mathrm{m} \varepsilon_{0}$,

$\omega_{p}$ - Plasmon frequency, $\mathrm{n}$ - electron number density, e - electron

charge, $\mathrm{m}^{-}$electron mass. 


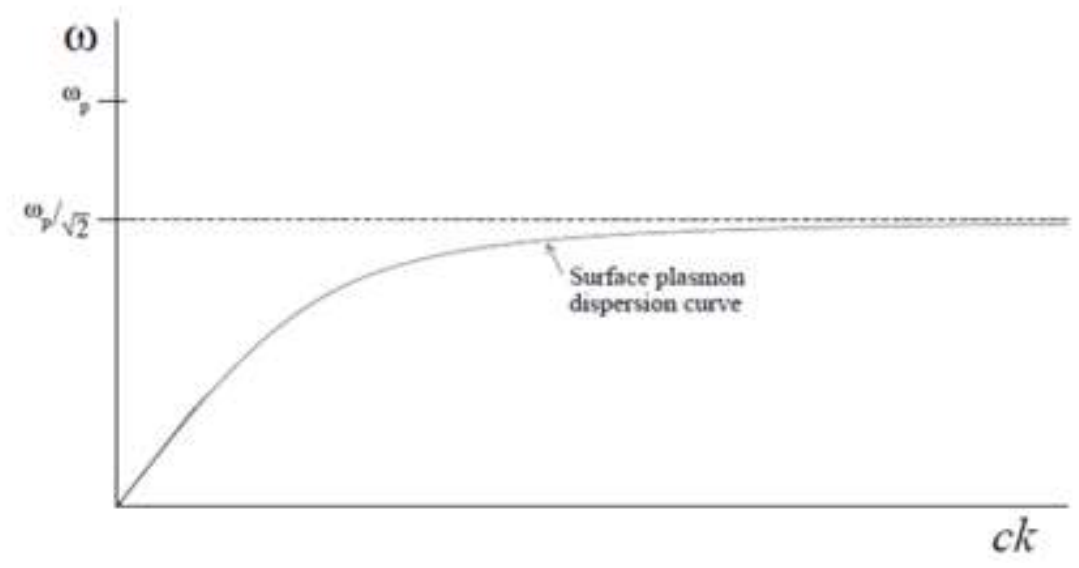

Figure 3. Dispersion Curve for SPR

In Figure 3, the lower frequency range the SPP wave number is near to the line light. Accordingly the surface wave extends deep into the dielectric material. For large frequencies the wave number becomes extremely large and tends to infinity as the resonance frequency is approached. This indicates the propagation speed slow down. At the same time, the mode is bounded close to the metal-dielectric surface as the frequency approach the resonance frequency $\omega_{\mathrm{p}}$ is given by

$$
\omega_{s p p}=\frac{\omega_{p}}{\sqrt{1+\varepsilon_{1}}}
$$

For surface plasmon excitation by incident light, Equation 5 conditions to be satisfied.

$$
K_{x}=\left(\frac{2 \pi}{\lambda}\right) n \sin \theta_{i}=K_{s p}=\left(\frac{2 \pi}{\lambda}\right) \sqrt{\frac{\varepsilon_{d} \varepsilon_{m}}{\varepsilon_{d}+\varepsilon_{m}}}
$$

p-polarized light to be incident from dielectric medium to metal and dielectric and metal must satisfy $\varepsilon_{\mathrm{m}^{\prime}}<0$ and $\left|\varepsilon_{\mathrm{m}^{\prime}}\right|>>\varepsilon_{\mathrm{m}^{\prime \prime}}$, Where

$\varepsilon_{\mathrm{m}}=\varepsilon_{\mathrm{m}^{\prime}}+\varepsilon_{\mathrm{m}^{\prime \prime}}$

Gold, Silver and Aluminium satisfy these conditions, hence gold was considered for design in present work [6].

\section{Sensor Design and Simulation}

The structure was designed by using Finite Difference Time Domain (FDTD) solver in RSoft CAD tool. The primary design considerations were accuracy and sensitivity. Sensitivity [7], defined as the ratio of change in characteristic wavelength to the change in the RI of the sample. A Micro Ring Resonator (MRR) structure is considered. MRR is an array of waveguides with at least one is a closed loop coupled for light input and output. When light of the resonant wavelength is passed through the loop, it builds up an intensity over multiple round-trips due to constructive interference and exit through the output bus waveguide thus the optical ring resonator functions as a filter. Figure 4 shows the initial design dimensions of the MRR Sensor. 


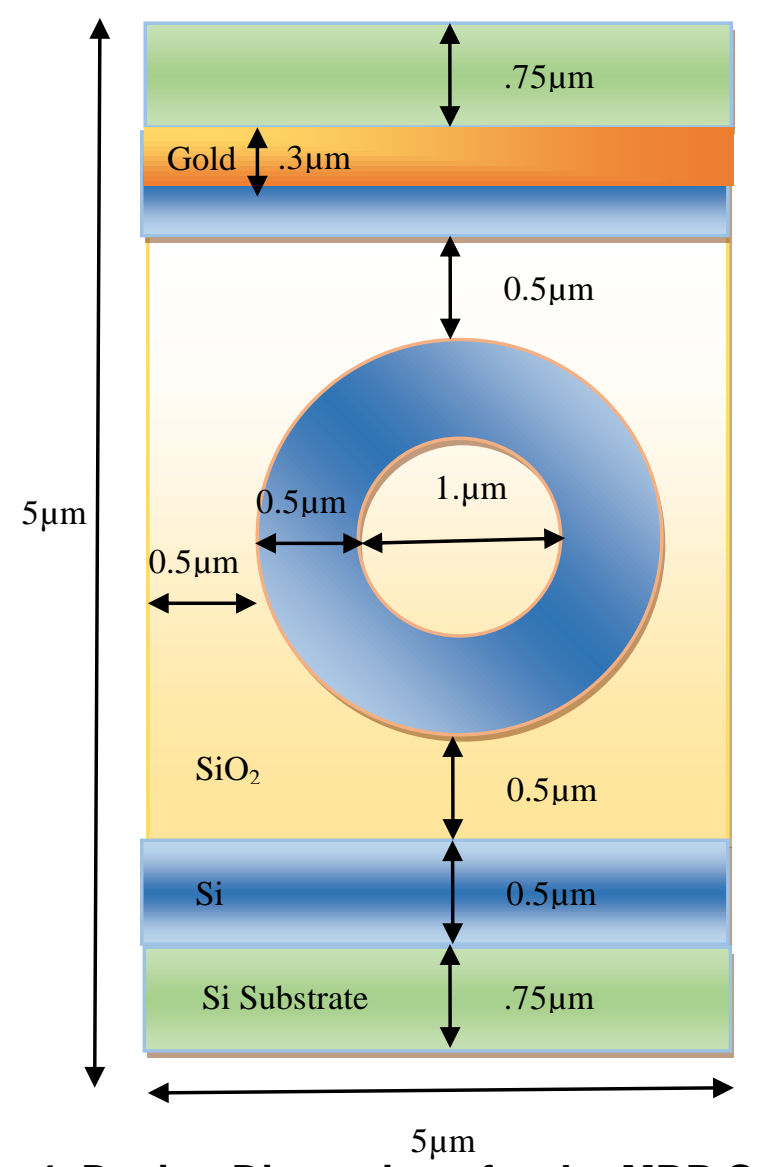

Figure 4. Design Dimensions for the MRR Sensor

The RI and thickness of the designed MRR is shown in Table 1.

Table 1. Design Parameters for SPR Based Sensor Structures

\begin{tabular}{|lrr|}
\hline \multicolumn{1}{|c}{ Material } & & Thickness in $\mu \mathrm{m}$ \\
\hline Silicon substrate & 3.45 & 1.5 \\
\hline Sio2 & 1.55 & 3 \\
\hline Si(ring resonator) & 3.45 & 2 \\
\hline Si (Bus) & 3.45 & 1 \\
\hline Gold & 0.5744 & 0.3 \\
\hline
\end{tabular}

The Figure 5 depicts the top view of the design, it consists of two layers, the bottom most layer is the silicon substrate and the second layer is the $\mathrm{SiO}_{2}$ layer in which Si ring resonator is embedded and one of the bus waveguides are coated with thin gold layer in uniform fashion across the length. A channel is provide beside the gold plated bus waveguide so as to place sample of interest. 


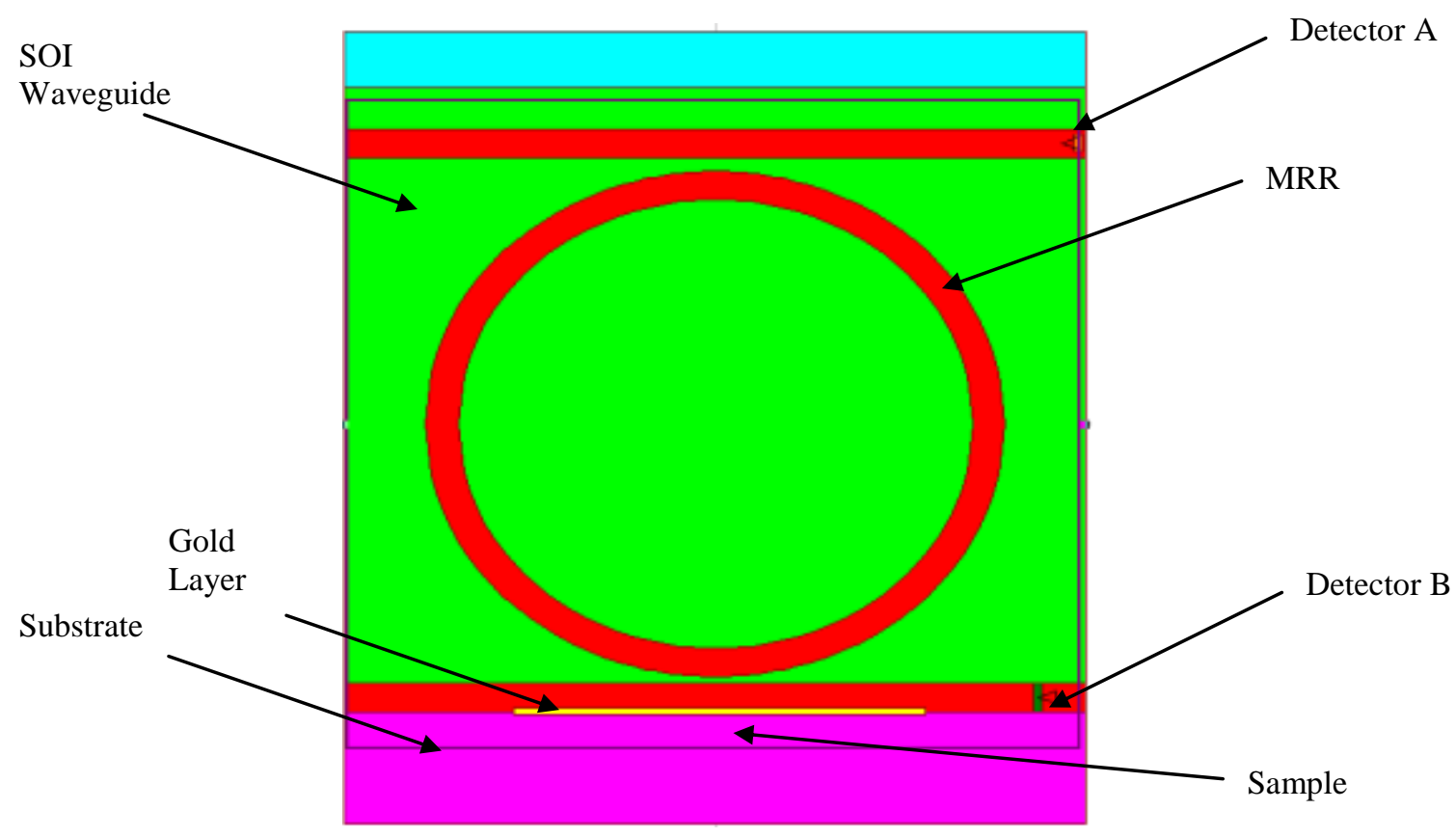

Figure 5. Top View of the Design as Seen in RSoft

We have optimized a simple MRR optical waveguide where gold is taken as metal layer. This structure involves an interferometer which consists of a silicon-on-insulator (SOI) waveguide that acts like a Mach-Zehnder [8]. It can be represented as metaldielectric interface as shown in the Figure 5. Reported sensitivity value for Mach-Zehnder interferometer is $250 \mathrm{~nm} / \mathrm{RIU}$ [9] and we aim to get significantly higher sensitivity than this [13].

The designed sensor is used to test in the solution containing alcohol, heroin and cocaine [10]. A broadband transverse magnetic (TM) light is made to pass through the input section and the entire system characteristics of the transmission spectrum is monitored by changing the RI of the sample medium [11]. The Gaussian pulse of laser was used to excite the structure and a monitor is placed at the end of the input (Detector A) and output (Detector B) section to observe the intensity of the transmitted light source. Two RI values 9.88 (Heroin), 2.21 (Cocaine), Alcohol (2.25) and Water (2.223) were considered and its output was correlated with that of the power at the end of the sensing section. Output wavelength observed in the range $2.05 \mu \mathrm{m}$ to $2.45 \mu \mathrm{m}$. Once the sensitivity is optimized, the sensor is used to detect drugs in alcohol. 


\section{Result and Analysis}

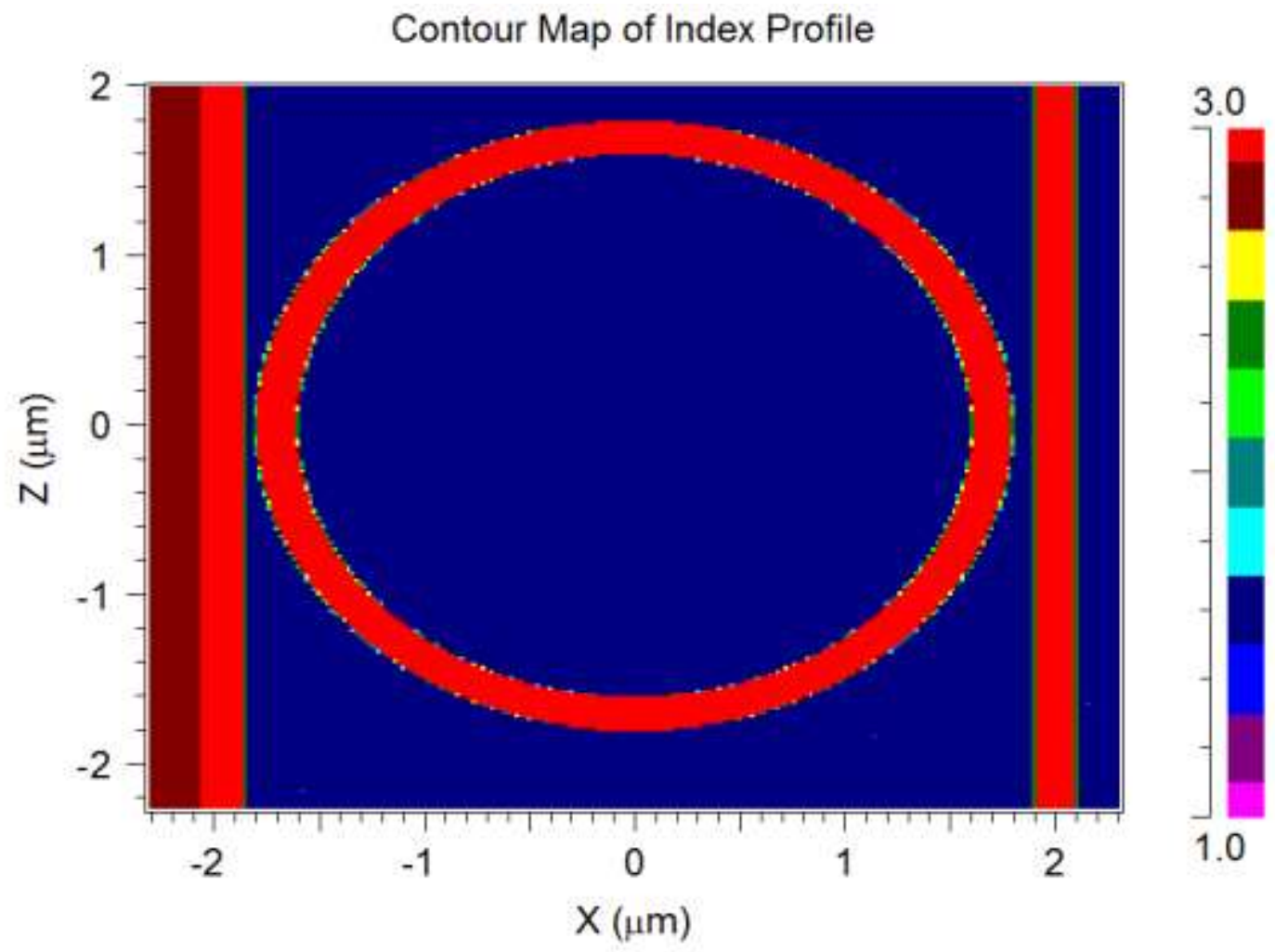

Figure 6. Contour Map of the MRR Sensor Structure

In Figure 6 the contour map of the structure represented, where different colour represents the refractive indices of different materials. This shows the correctness of the device. The range of refractive indices are defined in the colour scale given at the right side of the picture.

The Figure 7 (a) depicts the excitation inside the resonator with colour bar. 


\section{Contour Map of Ey}

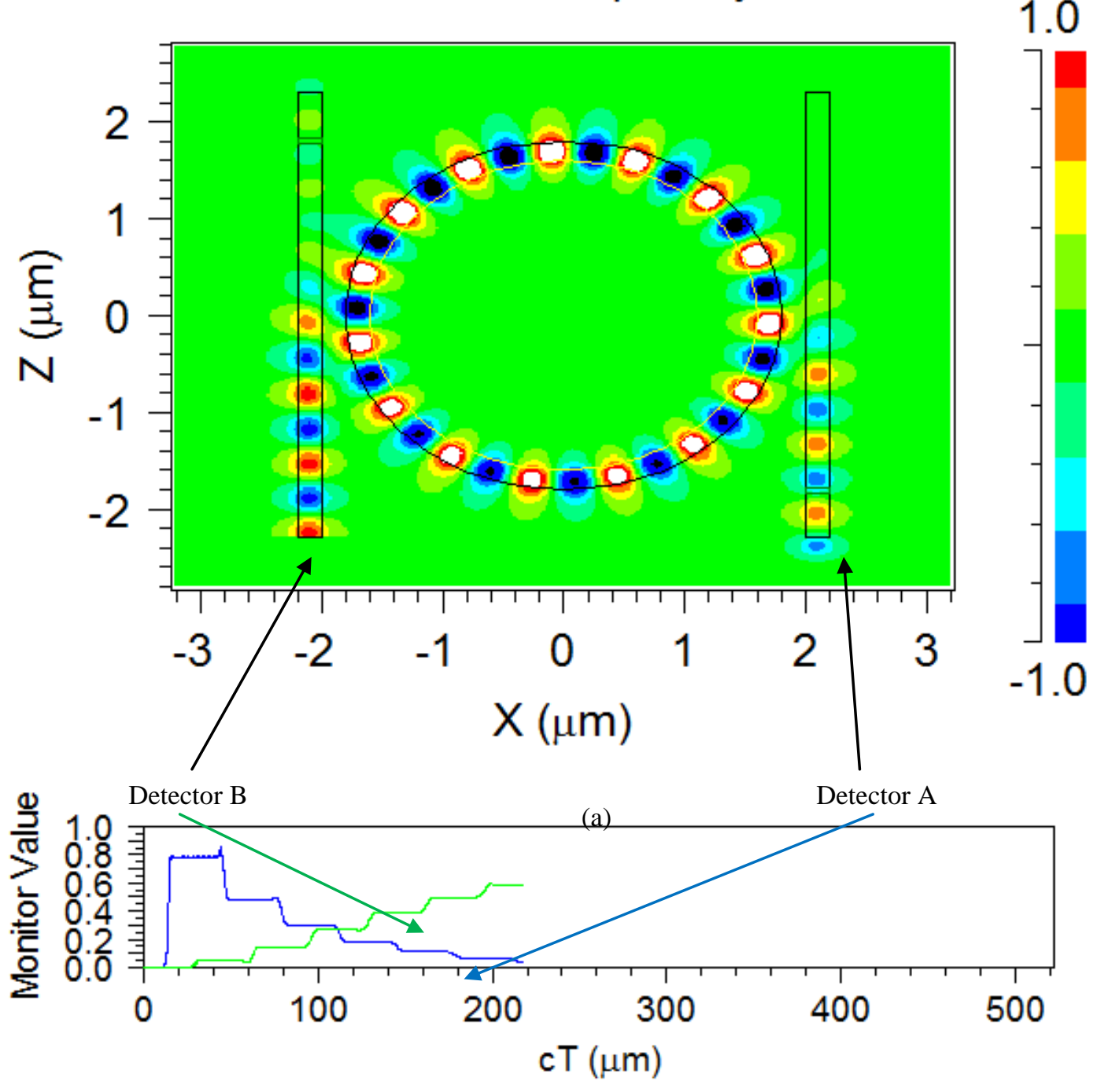

(b)

\section{Figure 7 (a) The Excitation Inside the MRR Sensor with Values Shown in the Color Bar at the (b) Instantaneous Monitor Behavior}

Figure 7 (b) Represents the instantaneous monitor behavior. The intensity of transmitted light gets diminished as shown in blue line as captured in detector A. The intensity of transmitted light gets enhanced as shown in green line as captured in detector B. The surface of gold (test bed) on a glass of a narrow flow path through which an aqueous (important to match RI) solution is continuously passed. In order to detect the binding of an analyte molecule to a receptor molecule, the receptor molecule is usually immobilised on the sensor surface and the analyte molecule is injected in the aqueous solution through the flow cell. Polarised light under the surface of the gold layer where surface plasmons are generated at a critical angle of the incident light. The power monitor was placed at the output (Detector B), the sudden increase and decrease (variation) in the strength of the signal is due to the constructive and destructive interference of the light. The decrement in the signal is due to the destructive interference of the light and increase in intensity is due to the constructive interference and this depends on the direction of light meeting at the output port or the region of interest. SPR intensity simulation result in the form of monitor power vs travelled distance over the gold plate is shown in Figure 8. 


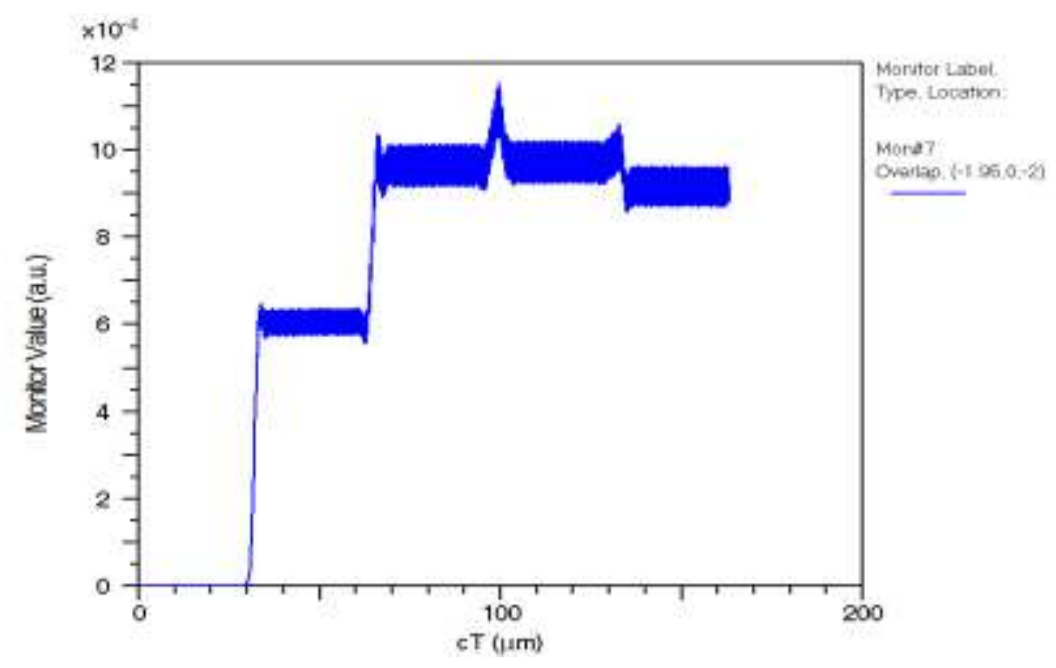

Figure 8. Monitor Power w.r.t Linear Distance Travelled by the Light along the Gold Plate

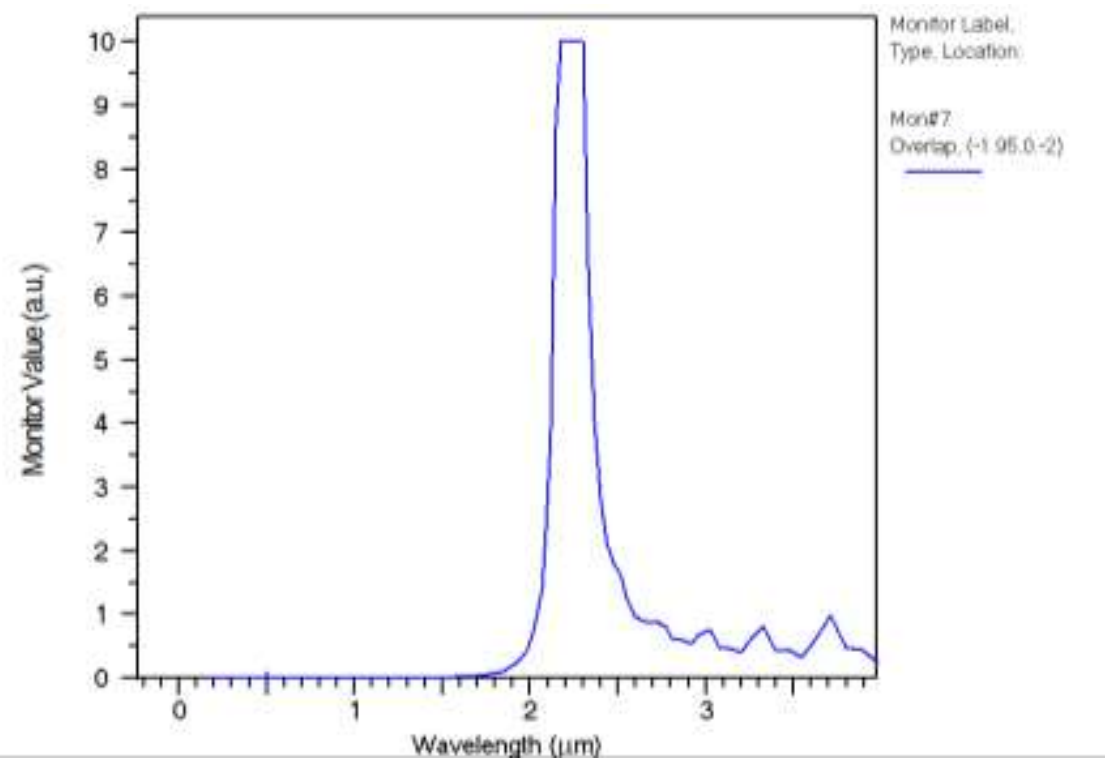

Figure 9. Wavelength Response of the Device at the Output Port B

The Figure 9 shows the resonance wavelength of the designed MRR. It is observed that the maximum intensity is at wavelength $2.55 \mu \mathrm{m}$ thus showing the filter operation is in action. That is for a critical angle of incident light the output intensity is maximum. 


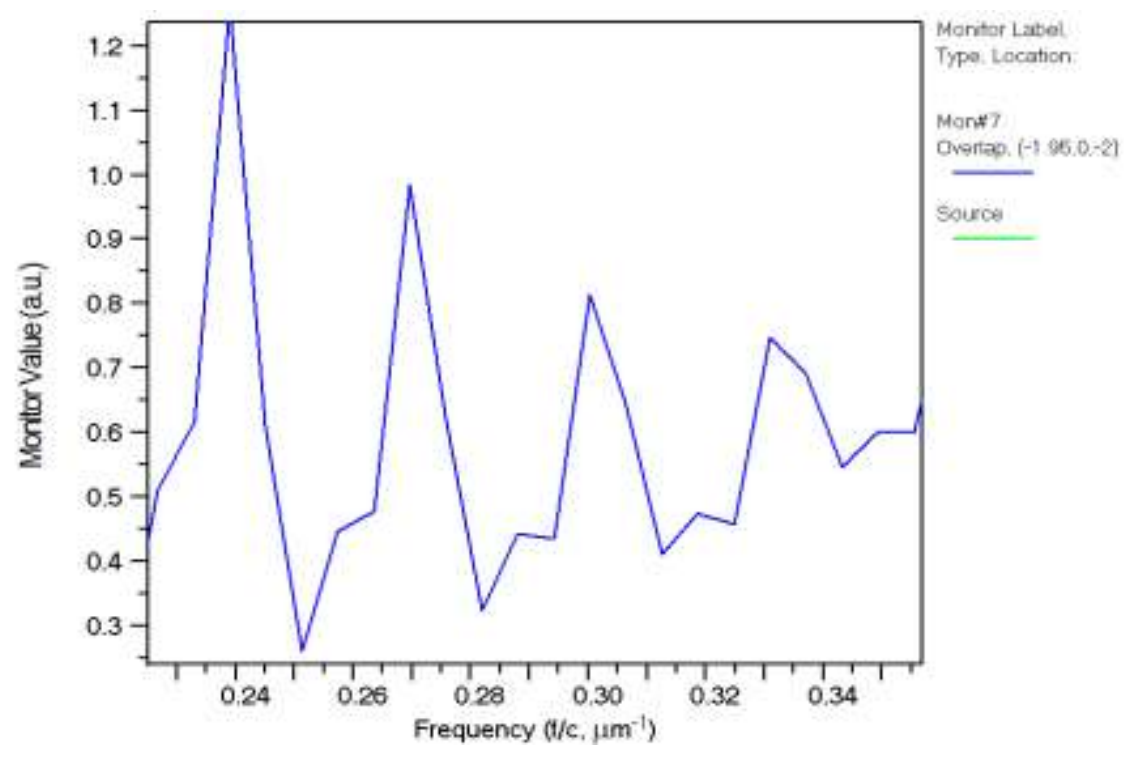

Figure 10. Monitor Output w r t Frequency at Output Port B

The graph in Figure 10 potrays the output power variation of the device with respect to frequency variation at the output port. The intensity is high where the frequency verifies the resonance region (as per Equation 4).

Table 2. Wavelength vs Normalized Intensity Obtained

\begin{tabular}{|c|c|c|}
\hline $\begin{array}{c}\text { Aqueous Solution } \\
\text { Ingredients }\end{array}$ & $\begin{array}{c}\text { Wavelength in } \mu \mathrm{m} \text { (taken } \\
\text { average) }\end{array}$ & $\begin{array}{c}\text { Maximum (Normalized } \\
\text { intensity) }\end{array}$ \\
\hline Water & 2.223 & 0.2 \\
\hline Alcohol & 2.25 & 0.4 \\
\hline Cocaine & 2.21 & 3.8 \\
\hline Heroin & 9.8 & 2.28 \\
\hline
\end{tabular}

Figure 11 shows the wavelength behavior for different samples such as alcohol, alcohol with cocaine, alcohol with heroin and water. It is observed that there is an intensity shift for respective samples centered at peak resonance wavelength at $2.4 \mu \mathrm{m}$. This verifies that the designed device can be utilized as a sensor for drug analysis. 


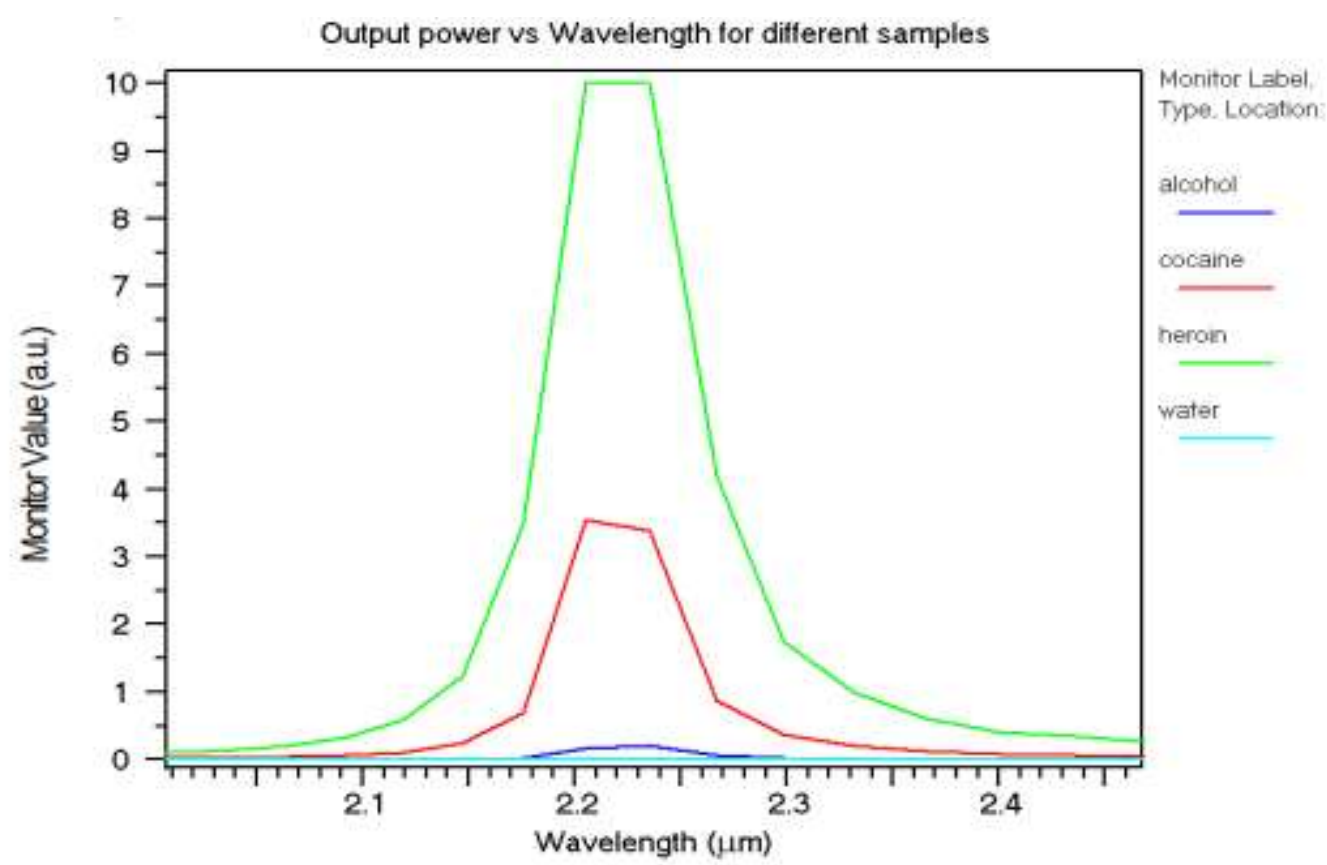

Figure 11. Output Power vs Wavelength for Different Samples

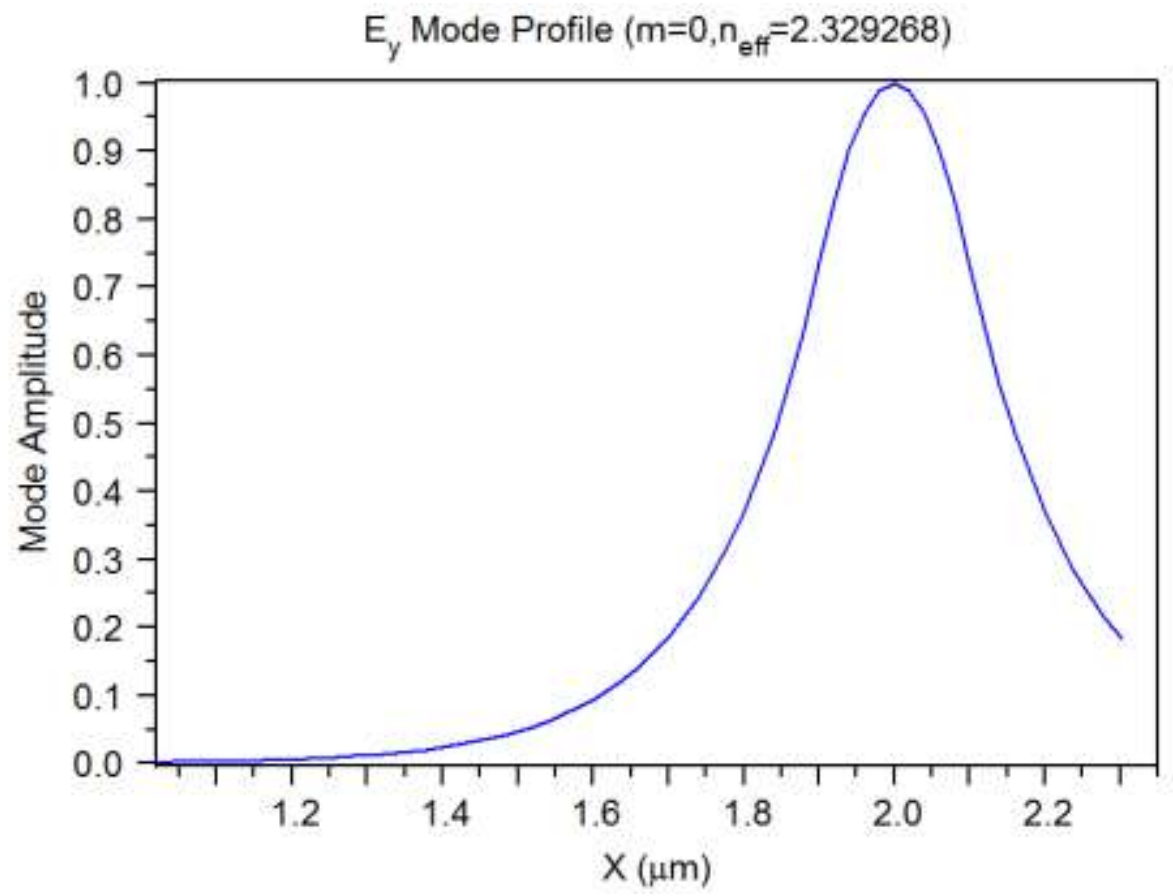

Figure 12. Launch Profile of the Device

The graph in Figure 12 shows the launch profile of the device corresponding to the position of the source. The $\mathrm{y}$ axis represents the mode amplitude and the $\mathrm{x}$ axis represents the layout dimensions for the device. It gives the effective RI calculation for the device materials at resonance wavelength and effective RI at resonance is 2.3292688 . 


\section{Conclusion}

In case of drug abuse, suspects use to mix non permissible drugs along with alcohol. We have designed the SPR based MRR for the detection of cocaine and heroin present in alcohol. The simulations are carried out for alcohol mixed with cocaine and heroin. The wavelength $\mathrm{w} r \mathrm{t}$ power graph has been plotted to analyse the variation or the behaviour of device at resonance wavelength i.e $2-2.55 \mu \mathrm{m}$. The device will allow only those wavelengths which will come within this range. Result shows that the intensity varies for cocaine and heroin. The intensity varies in accordance with the change in the RI value. The result obtained shows sensitivity of $3000 \mathrm{~nm} / \mathrm{RIU}$ and a Q factor of 2456 . The result is much higher than previously published result of $250 \mathrm{~nm} / \mathrm{RIU}$ for Mach-Zehnder type optical sensor implementation In future the analysis can be done with random concentration and more sample of drugs to be detected.

\section{References}

[1] Shalabney, A. and, Abdulhalim, I.: Sensitivity-enhancement methods for surface plasmon sensors, Laser \& Photonics Revs, Vol. 5 Issie 4, 571-606, (2011)

[2] Homola, J. Yee, S., Gauglitz, G.: Surface plasmon resonance sensors: review, Sens. Actuators B, vol. $54,3-15,(1999)$

[3] Toyama, S. et al..: Design and fabrication of a waveguide-coupled prism device for surface plasmon resonance sensor, Sens. Actuators B: Chemical, Vol. 65, 32-34, (2000).

[4] Hoa, X. D., Kirk, A. G., Tabrizian, M.: Towards integrated and sensitive surface plasmon resonance biosensors: A review of recent progress, Biosensors \& Bioelectronics, Vol. 23, 51-160, (2007).

[5] Ordal, M.A., Long, L.L., Bell, R.J., Bell, S.E., Bell, R.R., Alexander, R., Ward, J., Ward, C.A. "Optical properties of metals $\mathrm{Al}, \mathrm{Co}, \mathrm{Cu}, \mathrm{Au}, \mathrm{Fe}, \mathrm{Pb}, \mathrm{Ni}, \mathrm{Pd}, \mathrm{Pt}, \mathrm{Ag}, \mathrm{Ti}$, and Win the infrared and far infrared", Appl. Opt., Vol. 11, 1099-1119, (1983).

[6] Sun, Y. and Xia, Y.: Increased sensitivity of surface plasmon resonance of gold nano shells compared to that of gold solid colloids in response to environmental changes, Anal. Chem., Vol. 74, 5297-5305, (2002).

[7] Yu, X.L., Wang, D.X., Yan, Z.B.: Simulation and analysis of surface plasmon resonance biosensor based on phase detection, Sensors and Actuators, Vol. 91, 285-290, (2003)

[8] Debackere, P., Scheerlinck, S., Bienstman, P., Baets, R.: Surface plasmon interferometer in silicon-on insulator: novel concept for an integrated biosensor, Optics Express, Vol. 14, 7063-7072, (2006).

[9] Sookyoung, R. Taerin, C., Byoungho, L.: Overview of the Characteristics of Micro- and NanoStructured Surface Plasmon Resonance Sensor, Sensors, doi:10.90/s110201565, 1565 -1588, (2016)

[10] Homola, J., Vaisocherová, H., Dostálek, J., Piliarik, M.: Multi-analyte surface plasmon resonance biosensing, Methods, Vol. 37(1), 26-36, (2005).

[11] Hammond, J. L., Bhalla, N., Rafiee, S. D., Estrela, P.: Localized Surface Plasmon Resonance as a Biosensing Platform for Developing Countries, Biosensors, Vol. 4, 172-188, (2014).

[12] Danese, G., Leporati, F., Marabellia, F. : Localized Surface Plasmon Resonance-Based Portable Instrument for Quick On-Site Biomolecular Detection, IEEE Transactions on Instrumentation and Measurement, Vol. 65(2), 317 - 327, (2016).

[13] Sookyoung, R. Taerin, C., Byoungho, L.," Overview of the Characteristics of Micro- and NanoStructured Surface Plasmon Resonance Sensor", Sensors, 1565 -1588, (2016)

[14] M.A. Cooper, Advances in membrane receptor screening and analysis, J. Mol. Recognit. 17, 286-315, (2004).

[15] M. Besenicar, P. Macek, J.H. Lakey, G. Anderluh, Surface plasmon resonance in protein-membrane interactions, Chem. Phys. Lipids 141,169-178, (2006).

[16] Z. Salamon, H.A. Macleod, G. Tollin, Surface plasmon resonance spectroscopy as a tool for investigating the biochemical and biophysical properties of membrane protein systems. I: Theoretical principles, Biochim. Biophys. Acta-Reviews on Biomembranes 1331, 117-129, (1997).

[17] K. Kurihara, K. Suzuki, Theoretical understanding of an absorption-based surface plasmon resonance sensor based on Kretchmann's theory, Anal. Chem. 74, 696-701, (2002).

[18] J. Homola, Present and future of surface plasmon resonance biosensors, Anal. Bioanal. Chem. 377528 539, (2003).

[19] M. Raghavan, P.J. Bjorkman, BIAcore: a microchip-based system for analyzing the formation of macromolecular complexes, Structure 3, 331-333, (1995).

[20] L. Nieba, S.E. Nieba-Axmann, A. Persson, M. Hämäläinen, F. Edebratt, A. Hansson, J. Lidholm, K. Magnusson, A.F. Karlsson, A. Plückthun, BIACORE analysis of histidine-tagged proteins using a chelating NTA sensor chip, Anal. Biochem. 252 217-228, (1997).

[21] M. Malmqvist, BIACORE: an affinity biosensor system for characterization of biomolecular interactions, Biochem. Soc. Trans. 27, 335-340, (1999). 
[22] E. Schepens, S. Inman, B. J. McCullough, C. Hopley, Rapid confirmation and quantitation of drugs-ofabuse in oral fluid using a low cost, small footprint mass spectrometer, Forensic Chemistry 4, 75-81, (2017).

[23] Jahanshahi, P., Honarvar, B., Gang, S. S. Y., Adikan, F. R. M.: Numerical Analysis of SPR-Based Optical Fiber Biosensor", IEEE 5th International Conference on Photonics (ICP), 182-183, (2014).

[24] B, Juff, et al., Integrated Optical Mach-Zehnder Biosensor, Journal of Lightwave Technology, Vol. 16, pp. 583-592, (1998)

[25] www.drugabuse.gov/related-topics/trends-statistics\#supplemental-references-for-economic-costs

[26] www.surgeongeneral.gov/library/reports/50-years-of-progress/full-report.pdf

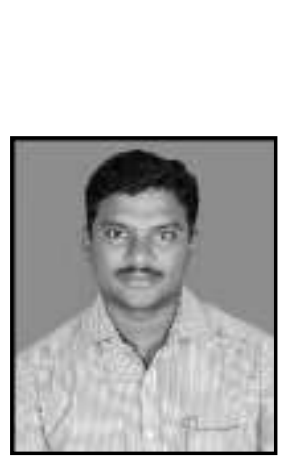

\section{Authors}

A Raganna is working as an Assistant Professor in the School of Electronics and Communication Engineering, REVA University, and pursuing his $\mathrm{PhD}$ in optical sensors. He has completed M.E in Microelectronic Systems from Indian Institute of Science (IISc) Bangalore and B.Tech. in Electronics and Communication Engineering from Jawaharlal Nehru Technological University (JNTUH) Hyderabad. He has 8 years of Teaching and Research experience and, 5 years Industrial Experience. He has over 9 publications in International journals and conferences. His research interests include Photonics, Bio-Sensors, Optical sensors, MEMS, NEMS, VLSI Technology etc.

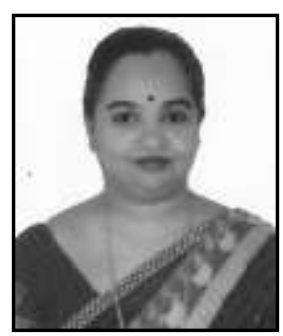

Bharathi S $\mathbf{H}$ is working as a Professor in the School of Electronics and Communication Engineering, REVA University. She has 25 years of teaching and research experience. She has many research publications in reputed national/international journals and conferences. She is involved in research in the area of research interests includes Photonics, Bio-Sensors, Optical sensors, Electromagnetism, Antenna, MEMS, NEMS Technology, Video processing, FPGA and, Currently, she is guiding $7 \mathrm{Ph}$. D. students. She is a member of IEEE and Women in Engineering (WIE).

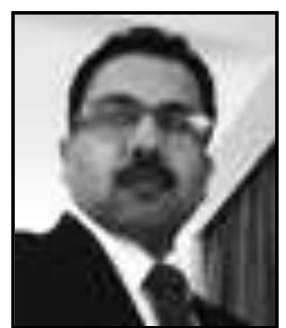

Sandip Kumar Roy, perusing $\mathrm{PhD}$ and interacting with leading research groups in his domain worldwide. Educated from primer institutes of India like BHU, IIT to create the foundation for technical understanding and instill focus on innovation through research in work. Visited facilities of leading universities across world like Texas Tech USA, NTU Singapore, Tohoku University Japan and University of Macau, China. Actively involved in resesrch for $\mathrm{PhD}$ work in Photonics, Optical Sensor, GPS, Embedded systems, Human Computer Interaction and Smart Health Monitoring. Working on human computer interaction and the Smart Device for Persons with Disability to Interact with Computer bagged 1st award for design in 2015. Patent filed as part of interface requirements for sensor being designed part of $\mathrm{PhD}$ work. More than 15 publications (majority Scopus indexed). Presented research work and attended many prestigious conferences worldwide in Malaysia, USA, Japan, Macau, Singapore. 


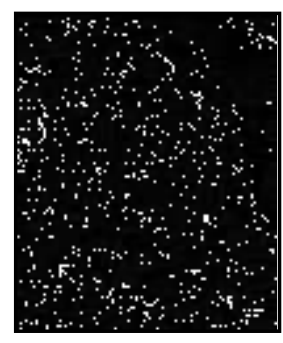

Preeta Sharan, a post doctorate from IIT Kharagpur and currently a competent professor with $22+$ years of experience in Education, Research, Student Mentorship, association with international universities and organizations. Responsible for teaching B.Tech/ M.Tech. Guide Phd. students and execute government/ international agency funded projects. A self-starter with strong entrepreneurial spirit, built R\&D center of my own with team size $15+$ from scratch. I have delivered projects for prestigious funding agencies across the world as lead investigator like Naval Research Board, DRDO, India, IEEE USA and Govt. of Karnataka (KVGST). With multiple patents in process, research publications reaching $100+$ under my leadership. Visited facilities of leading universities NTU Singapore, Tohoku University, Japan and University of Macau. Published papers in premier journals and presented papers in IEEE conferences in Malaysia, Singapore, Japan and Macau.

Awarded "Woman of the Year - 1998, 2009" by American Biographical Institute. Associated with Elsevier and McGraw hill publication as reviewer. 